\title{
Integration of Fenchel Subdifferentials Revisited
}

\author{
Juan Enrique Martínez-Legaz \\ Dedicated to Professor Boris Mordukhovich on his 65th birthday
}

Received: 20 September 2013 / Accepted: 20 June 2014

\begin{abstract}
We obtain a simple integration formula for Fenchel subdifferentials on Euclidean spaces and analyze some of its consequences. For functions defined on locally convex spaces, we present a similar result in terms of $\varepsilon$-subdifferentials.
\end{abstract}

Keywords Convex functions $\cdot$ Fenchel subdifferential $\cdot$ Rockafellar integration formula

Mathematics Subject Classification (2010) $26 \mathrm{~B} 25$

\section{Introduction}

The integration of Fenchel subdifferentials, that is, the problem of recovering a convex function from its associated Fenchel subdifferential mapping, is a fundamental problem in Convex Analysis. Its classical solution is provided by the so called Rockafellar integration formula, which is implicit in the proof of Theorem 1 of [11]. An alternative integration formula was given by A. Verona and M.E. Verona [14, Lemma 2.4]. In [1, Proposition 27], M. Bachir, A. Daniilidis and J.-P. Penot proved that Rockafellar integration formula applied to a nonconvex function $f$ yields the second Fenchel conjugate $f^{* *}$, provided that $f$ is lower semicontinuous (1.s.c., in short) and 1-coercive. This result was extended to the larger class of epi-pointed functions by J. Benoist and A. Daniilidis [2, Theorem 3.5], who also proved its validity for arbitrary 1.s.c. one variable functions [2, Corollary 3.7]. Later on these authors refined Rockafellar formula for the same class of functions, by proving that one can

This research was supported by the MICINN of Spain, Grant MTM2011-29064-C03-01, and under Australian Research Council's Discovery Projects funding scheme (project number DP140103213). The author is affiliated to MOVE (Markets, Organizations and Votes in Economics).

J.E. Martínez-Legaz (ه)

Departament d'Economia i d'Història Econòmica, Universitat Autònoma de Barcelona, 08193 Bellaterra, Spain

E-mail: JuanEnrique.Martinez.Legaz@uab.cat

$$
\begin{aligned}
& \text { This is the accepted version of the article published by Springer Science } \\
& \text { +Business Media: Martínez-Legaz, J.E., (2014) Integration of Fenchel } \\
& \text { Subdifferentials Revisited, Vietnam Journal of Mathematics, } 42 \text { (2): p.533-542. } \\
& \text { The final version is available at: https://doi.org/10.1007/s10013-014-0101-3 }
\end{aligned}
$$


restrict the supremum in formula (7) below to points $x_{i}$ such that $\left(x_{i}, f\left(x_{i}\right)\right)$ are exposed points in the epigraph of $f$, even in the case when $f$ is defined on an infinite dimensional Banach space with the Radon-Nikodym property [3, Theorem 3.7]. Another integration result for epi-pointed nonconvex functions on Banach spaces, in terms of $\varepsilon$-subdifferentials, has been recently obtained by R. Correa, Y. García and A. Hantoute [5, Theorem 13]. Finally, a variant of this result, valid for nonnecessarily epi-pointed functions on general normed spaces, is due to M.A. López and M. Volle [8, Theorem 4].

In this paper we obtain a very simple integration result for convex functions on $\mathbb{R}^{n}$, in terms of Fenchel subdifferentials, by means of a quite elementary yet powerful technique devised by P. Kocourek [7, Proof of Theorem 1], who used it to provide a simple proof of Corollary 2 below, employed also in [6] to characterize Lipschitz DC functions in terms of $\varepsilon$-subdifferentials. From our new result, Rockafellar integration formula follows as an immediate corollary, but the two formulas are significantly different. Our formula can be expressed by means of a supremum, like in the case of Rockafellar formula, but also as an ordinary limit, and does not require the knowledge of the whole subdifferential mapping but just of an arbitrary single valued selection of this mapping. We present a detailed analysis of the new formula and draw several of its consequences. For functions defined on general locally convex spaces, we present an alternative integration formula involving $\varepsilon$-subdifferentials.

All the convex analytic notions, notations and results we will use are fairly standard. We refer to the classical book [12] for finite dimensional Convex Analysis, and to [15] for an excellent treatment of its extension to the setting of locally convex spaces.

The rest of the paper consists of three sections. In Section 2 we present and analyze the new integration formula for Fenchel subdifferentials of 1.s.c. convex functions defined on $\mathbb{R}^{n}$, and discuss some of its consequences. Using the new integration formula, in Section 3 we obtain a characterization of $\varepsilon$-subgradients in terms of subgradients, which is considerably simpler than that of [9, Theorem 1]. Section 4 deals with the general locally convex case, in which the difficulty caused by the absence of a suitable result on the nonemptiness of the Fenchel subdifferential is overcome by considering $\varepsilon$-subdifferentials.

\section{Integration of Fenchel Subdifferentials of Functions Defined on $\mathbb{R}^{n}$}

In this section we consider 1.s.c. convex functions defined on $\mathbb{R}^{n}$. The main result, which is presented next, is a new integration formula for Fenchel subdifferentials.

Theorem 1 Let $f: \mathbb{R}^{n} \longrightarrow \mathbb{R} \cup\{+\infty\}$ be a l.s.c. convex function, $x_{0} \in \operatorname{ri} \operatorname{dom} f$, and $s: \operatorname{ridom} f \longrightarrow \mathbb{R}^{n}$ be a selection of the subdifferential mapping $\partial f$. For $x \in \operatorname{cl} \operatorname{dom} f$, 
$m=2,3, \ldots$ and $i=0,1, \ldots, m$, denote $x_{m, i}:=x_{0}+\frac{i}{m}\left(x-x_{0}\right)$. Then

$$
\begin{aligned}
f(x) & =f\left(x_{0}\right)+\lim _{m \rightarrow \infty} \frac{1}{m}\left\langle x-x_{0}, \sum_{i=0}^{m-1} s\left(x_{m, i}\right)\right\rangle \\
& =f\left(x_{0}\right)+\sup _{m} \frac{1}{m}\left\langle x-x_{0}, \sum_{i=0}^{m-1} s\left(x_{m, i}\right)\right\rangle \quad \forall x \in \operatorname{cldom} f .
\end{aligned}
$$

Proof We have

$$
f\left(x_{m, i+1}\right)-f\left(x_{m, i}\right) \geq \frac{1}{m}\left\langle x-x_{0}, s\left(x_{m, i}\right)\right\rangle \text { for } i=0,1, \ldots, m-1 .
$$

Adding up these inequalities, we get

$$
f(x)-f\left(x_{0}\right) \geq \frac{1}{m}\left\langle x-x_{0}, \sum_{i=0}^{m-1} s\left(x_{m, i}\right)\right\rangle,
$$

and hence

$$
f(x)-f\left(x_{0}\right) \geq \varlimsup_{m \rightarrow \infty} \frac{1}{m}\left\langle x-x_{0}, \sum_{i=0}^{m-1} s\left(x_{m, i}\right)\right\rangle .
$$

On the other hand, we also have

$$
f\left(x_{m, i-1}\right)-f\left(x_{m, i}\right) \geq-\frac{1}{m}\left\langle x-x_{0}, s\left(x_{m, i}\right)\right\rangle \quad \text { for } i=1, \ldots, m-1,
$$

and adding up these inequalities we get

$$
f\left(x_{0}\right)-f\left(x_{m, m-1}\right) \geq-\frac{1}{m}\left\langle x-x_{0}, \sum_{i=1}^{m-1} s\left(x_{m, i}\right)\right\rangle
$$

therefore, since $\underline{\lim }_{m \rightarrow \infty} f\left(x_{m, m-1}\right) \geq f(x)$ due to the lower semicontinuity of $f$,

$$
\begin{aligned}
f\left(x_{0}\right)-f(x) & \geq-\varliminf_{m \rightarrow \infty} \frac{1}{m}\left\langle x-x_{0}, \sum_{i=1}^{m-1} s\left(x_{m, i}\right)\right\rangle \\
& =-\varliminf_{m \rightarrow \infty} \frac{1}{m}\left(\left\langle x-x_{0}, \sum_{i=0}^{m-1} s\left(x_{m, i}\right)\right\rangle-\left\langle x-x_{0}, s\left(x_{m, 0}\right)\right\rangle\right) \\
& =-\varliminf_{m \rightarrow \infty} \frac{1}{m}\left\langle x-x_{0}, \sum_{i=0}^{m-1} s\left(x_{m, i}\right)\right\rangle
\end{aligned}
$$

From (3) and (6), equality (1) immediately follows.

The classical Rockafellar integration formula is an immediate consequence of Theorem 1: 
Corollary 1 [11] Let $f$ and $x_{0}$ be as in Theorem 1 . Then, for every $x \in \operatorname{cl} \operatorname{dom} f$ one has

$$
f(x)=f\left(x_{0}\right)+\sup _{\substack{\left(x_{i}, x_{i}^{*}\right) \in \operatorname{graph} \partial f \\(i=1, \ldots, m)}}\left\{\sum_{i=0}^{m-1}\left\langle x_{i+1}-x_{i}, x_{i}^{*}\right\rangle+\left\langle x-x_{m}, x_{m}^{*}\right\rangle\right\} .
$$

Proof The inequality " $\geq$ " in (7) easily follows from the subgradient inequality. To prove the opposite inequality, use Theorem 1 after setting $x_{i}:=x_{m+1, i}$ and $x_{i}^{*}:=$ $s\left(x_{m+1, i}\right)$ in (7), which yields

$$
\sum_{i=0}^{m-1}\left\langle x_{i+1}-x_{i}, x_{i}^{*}\right\rangle+\left\langle x-x_{m}, x_{m}^{*}\right\rangle=\frac{1}{m+1}\left\langle x-x_{0}, \sum_{i=0}^{m} s\left(x_{m+1, i}\right)\right\rangle .
$$

The following result is an easy consequence of Theorem 1 too.

Corollary 2 [7, Corollary 1] Let $f, g: \mathbb{R}^{n} \longrightarrow \mathbb{R} \cup\{+\infty\}$ be l.s.c. convex functions with $\mathrm{cl} \operatorname{Dom} \partial f=\mathrm{cl} \operatorname{Dom} \partial g=: C$ and

$$
\partial f(x) \cap \partial g(x) \neq \emptyset \quad \forall x \in \operatorname{riC} .
$$

Then $f=g+$ const.

Proof We have ri $\operatorname{dom} f \subseteq \operatorname{Dom} \partial f \subseteq \operatorname{dom} f$ (see [12, Theorem 23.4]), from which one can easily deduce that $\mathrm{cl} \operatorname{dom} f=C$, and hence $\operatorname{ri} \operatorname{dom} f=\operatorname{ri} C$. In the same way, we have cl $\operatorname{dom} g=C$; by [12, Theorem 6.3], from this equality it follows that ri $\operatorname{dom} g=\operatorname{ri} C$. Then, for $x_{0} \in \operatorname{ri} C$, applying Theorem 1 with $s: \operatorname{ri} C \rightrightarrows \mathbb{R}^{n}$ such that $s(x) \in \partial f(x) \cap \partial g(x)$, we get

$$
f(x)=f\left(x_{0}\right)+\lim _{m \rightarrow \infty} \frac{1}{m}\left\langle x-x_{0}, \sum_{i=0}^{m-1} s\left(x_{m, i}\right)\right\rangle \quad \forall x \in C,
$$

and

$$
g(x)=g\left(x_{0}\right)+\lim _{m \rightarrow \infty} \frac{1}{m}\left\langle x-x_{0}, \sum_{i=0}^{m-1} s\left(x_{m, i}\right)\right\rangle \quad \forall x \in C,
$$

which shows that $f=g+f\left(x_{0}\right)-g\left(x_{0}\right)$.

It is worth mentioning that Corollary 2 also follows from the classical integration result ([11, Theorem 3], [12, Theorem 24.9]) that says that $\partial f=\partial g$ implies $f=g+$ const. Indeed, assuming, without loss of generality, that $C$ has a nonempty interior, condition (8) implies that the gradients of $f$ and $g$ coincide at every common differentiability point. Since the set of such points is dense in $C$ [12, Theorem 25.5] and, on the other hand, the subdifferential at a point is determined by the gradients at neighboring differentiability points, it turns out that condition (8) actually implies the equality $\partial f=\partial g$.

Our next result states the uniqueness, up to an additive constant, of the integral of a (nonnecessarily maximal) cyclically monotone mapping under the assumption that its domain has a convex relative interior. 
Corollary 3 Let $A: \mathbb{R}^{n} \rightrightarrows \mathbb{R}^{n}$ be cyclically monotone. If ri Dom $A$ is convex, then there exists a l.s.c. convex function $f: \operatorname{cl} \operatorname{Dom} A \longrightarrow \mathbb{R} \cup\{+\infty\}$ such that $A(x) \subseteq$ $\partial f(x)$ for every $x \in \mathbb{R}^{n}$, uniquely determined up to an additive constant.

Proof By [12, Theorem 24.8], there exists a l.s.c. convex function $f: \mathbb{R}^{n} \longrightarrow \mathbb{R} \cup$ $\{+\infty\}$ such that $A(x) \subseteq \partial f(x)$ for every $x \in \mathbb{R}^{n}$. We can assume, without loss of generality, that $\operatorname{cl} \operatorname{Dom} \partial f=\operatorname{cl} \operatorname{Dom} A$. Let $g$ be another function with the same properties as $f$, and take a single valued selection $s$ of $A$. Since $s(x) \in A(x) \subseteq \partial f(x) \cap \partial g(x)$ for every $x \in$ ri $\operatorname{Dom} A$, the conclusion follows from Corollary 2.

From Corollary 3 , the uniqueness part of the classical integration result for maximal cyclically monotone mappings easily follows:

Corollary $4\left[12\right.$, Theorem 24.9] Let $A: \mathbb{R}^{n} \rightrightarrows \mathbb{R}^{n}$ be maximal cyclically monotone. Then there exists a l.s.c. convex function $f: \operatorname{cl} \operatorname{Dom} A \longrightarrow \mathbb{R} \cup\{+\infty\}$ such that $A=$ $\partial f$, uniquely determined up to an additive constant.

Proof Combining the existence part of [12, Theorem 24.9] with [12, Corollary 31.5.2], we deduce that $A$ is maximal monotone. Hence, by a classical theorem of Minty [10] (see also [13, Theorem 12.41]), the set $\operatorname{Dom} A$ is nearly convex, that is, it contains the relative interior of its convex hull, which implies that this relative interior coincides with that of $\operatorname{Dom} A$. Therefore ri $\operatorname{Dom} A$ is convex, and thus Corollary 3 applies.

Our next result is another consequence of Corollary 3.

Corollary 5 Let $A: \mathbb{R}^{n} \rightrightarrows \mathbb{R}^{n}$ be cyclically monotone. If $\operatorname{Dom} A$ is convex and relatively open, then all the maximal cyclically monotone extensions of $A$ have the same restriction to $\operatorname{Dom} A$.

Proof Let $M_{1}$ and $M_{2}$ be two maximal cyclically monotone extensions of $A$. By [12, Theorem 24.9], there exist two 1.s.c. convex functions $f_{1}, f_{2}: \mathbb{R}^{n} \longrightarrow \mathbb{R} \cup\{+\infty\}$ such that $\partial f_{i}=M_{i}(1=1,2)$. By Corollary 3 , the restrictions of $f_{1}$ and $f_{2}$ to $\operatorname{cl} \operatorname{Dom} A$ coincide up to an additive constant. Hence, $\partial f_{1}(x)=\partial f_{2}(x)$ for every $x \in \operatorname{Dom} A$, which shows that $M_{1}$ and $M_{2}$ coincide on $\operatorname{Dom} A$.

Using the fact that the subdifferential of a convex function $f$ at a point in $\operatorname{ridom} f$ is the sum of a compact convex set with the orthogonal subspace to the affine hull of ri $\operatorname{dom} f$, and its support function is the directional derivative at that point, as a function of the direction, we obtain the following corollary of Theorem 1.

Corollary 6 Let $f$ and $x_{0}$ be as in Theorem 1 . Then

$$
\begin{aligned}
f(x) & =f\left(x_{0}\right)+\lim _{m \rightarrow \infty} \frac{1}{m} \sum_{i=0}^{m-1} f^{\prime}\left(x_{m, i}, x-x_{0}\right) \\
& =f\left(x_{0}\right)+\sup _{m} \frac{1}{m} \sum_{i=0}^{m-1} f^{\prime}\left(x_{m, i}, x-x_{0}\right) \\
& =f\left(x_{0}\right)-\lim _{m \rightarrow \infty} \frac{1}{m} \sum_{i=0}^{m-1} f^{\prime}\left(x_{m, i},-\left(x-x_{0}\right)\right) \\
& =f\left(x_{0}\right)-\inf _{m} \frac{1}{m} \sum_{i=0}^{m-1} f^{\prime}\left(x_{m, i},-\left(x-x_{0}\right)\right) \quad \forall x \in \operatorname{cldom} f .
\end{aligned}
$$


Proof Apply Theorem 1, first with $s\left(x_{m, i}\right)$ such that $\left\langle x-x_{0}, s\left(x_{m, i}\right)\right\rangle=f^{\prime}\left(x_{m, i}, x-x_{0}\right)$, and then with $s\left(x_{m, i}\right)$ such that $\left\langle x-x_{0}, s\left(x_{m, i}\right)\right\rangle=-f^{\prime}\left(x_{m, i},-\left(x-x_{0}\right)\right)$.

According to the next result, the supremum and the infimum in (9) are attained only in exceptional cases.

Proposition 1 Let $f, x_{0}$ and $s$ be as in Theorem 1. If the supremum or the infimum in (1) or (9) are attained, then the restriction of $f$ to the segment $\left[x_{0}, x\right]$ is polyhedral.

Proof We will just consider the case of (1), since the formulas in (9) are particular cases. Assume that the supremum is attained at $m$. In view of (2), we have

$$
f\left(x_{m, i+1}\right)-f\left(x_{m, i}\right)=\frac{1}{m}\left\langle x-x_{0}, s\left(x_{m, i}\right)\right\rangle \quad \text { for } i=0,1, \ldots, m-1 .
$$

Hence, for $i=0,1, \ldots, m-1$ and $t \in[0,1]$, we obtain

$$
\begin{aligned}
f\left(x_{m, i}\right)+t\left\langle x_{m, i+1}-x_{m, i}, s\left(x_{m, i}\right)\right\rangle & \leq f\left(x_{m, i}+t\left(x_{m, i+1}-x_{m, i}\right)\right) \\
& \leq f\left(x_{m, i}\right)+t\left(f\left(x_{m, i+1}\right)-f\left(x_{m, i}\right)\right) \\
& =f\left(x_{m, i}\right)+\frac{t}{m}\left\langle x-x_{0}, s\left(x_{m, i}\right)\right\rangle \\
& =f\left(x_{m, i}\right)+t\left\langle x_{m, i+1}-x_{m, i}, s\left(x_{m, i}\right)\right\rangle ;
\end{aligned}
$$

therefore $f\left(x_{m, i}+t\left(x_{m, i+1}-x_{m, i}\right)\right)=f\left(x_{m, i}\right)+t\left\langle x_{m, i+1}-x_{m, i}, s\left(x_{m, i}\right)\right\rangle$, which shows that the restriction of $f$ to the segment $\left[x_{m, i}, x_{m, i+1}\right]$ is affine.

Corollary 7 Let $f, x_{0}$ and $s$ be as in Theorem 1 . If $f$ is strictly convex then the supremum and the infimum in (1) and (9) are not attained.

The sequences $\left\{\frac{1}{m}\left\langle x-x_{0}, \sum_{i=0}^{m-1} s\left(x_{m, i}\right)\right\rangle\right\},\left\{\frac{1}{m} \sum_{i=0}^{m-1} f^{\prime}\left(x_{m, i}, x-x_{0}\right)\right\}$, and $\left\{\frac{1}{m} \sum_{i=0}^{m-1}\right.$ $\left.f^{\prime}\left(x_{m, i},-\left(x-x_{0}\right)\right)\right\}$ need not be monotone. Consider, for instance, the case when $n=1, f(x)=|x|, x_{0}=-1$ and $s$ is the selection of $\partial f$ such that $s(0)=1$; in this case, for $x=1$ a straightforward calculation yields

$$
\frac{1}{m}\left\langle x-x_{0}, \sum_{i=0}^{m-1} s\left(x_{m, i}\right)\right\rangle=\frac{1}{m} \sum_{i=0}^{m-1} f^{\prime}\left(x_{m, i}, x-x_{0}\right)=\frac{(-1)^{m}+1}{m} .
$$

However, it is easy to extract monotone subsequences, as the following proposition shows.

Proposition 2 Let $f, x_{0}$ and $s$ be as in Theorem 1. If $\left\{m_{k}\right\}$ is an increasing sequence of positive integer numbers with the property that $m_{k+1}$ is a multiple of $m_{k}$ for each $k$, then the subsequences $\left\{\frac{1}{m_{k}}\left\langle x-x_{0}, \sum_{i=0}^{m_{k}-1} s\left(x_{m_{k}, i}\right)\right\rangle\right\}$ and $\left\{\frac{1}{m_{k}} \sum_{i=0}^{m_{k}-1} f^{\prime}\left(x_{m_{k}, i}, x-x_{0}\right)\right\}$ are nondecreasing, and the subsequence $\left\{\frac{1}{m_{k}} \sum_{i=0}^{m_{k}-1} f^{\prime}\left(x_{m_{k}, i},-\left(x-x_{0}\right)\right)\right\}$ is nonincreasing. These monotonicity properties are strict if $f$ is strictly convex. 
Proof We prove the result only for the first subsequence. Setting $z_{k}:=\frac{m_{k+1}}{m_{k}}$, we get

$$
\begin{aligned}
\frac{1}{m_{k}}\left\langle x-x_{0}, \sum_{i=0}^{m_{k}-1} s\left(x_{m_{k}, i}\right)\right\rangle & =\frac{1}{m_{k}} \sum_{i=0}^{m_{k}-1}\left\langle x-x_{0}, s\left(x_{m_{k}, i}\right)\right\rangle \\
& \leq \frac{1}{m_{k}} \sum_{i=0}^{m_{k}-1} \frac{1}{z_{k}}\left\langle x-x_{0}, \sum_{j=i z_{k}}^{(i+1) z_{k}-1} s\left(x_{m_{k+1}, j}\right)\right\rangle \\
& =\frac{1}{m_{k} z_{k}} \sum_{i=0}^{m_{k}-1} \sum_{j=i z_{k}}^{(i+1) z_{k}-1}\left\langle x-x_{0}, s\left(x_{m_{k+1}, j}\right)\right\rangle \\
& =\frac{1}{m_{k+1}} \sum_{i=0}^{m_{k+1}-1}\left\langle x-x_{0}, s\left(x_{m_{k+1}, i}\right)\right\rangle \\
& =\frac{1}{m_{k+1}}\left\langle x-x_{0}, \sum_{i=0}^{m_{k+1}-1} s\left(x_{m_{k+1}, i}\right)\right\rangle .
\end{aligned}
$$

The preceding inequality follows from the monotonicity of the subdifferential mapping; indeed, for $j=i z_{k}+1, \ldots,(i+1) z_{k}-1$, we have

$$
\begin{aligned}
\left\langle x-x_{0}, s\left(x_{m_{k}, i}\right)-s\left(x_{m_{k+1}, j}\right)\right\rangle & =\left\langle x-x_{0}, s\left(x_{m_{k+1}, i z_{k}}\right)-s\left(x_{m_{k+1}, j}\right)\right\rangle \\
& =\frac{m_{k+1}}{j-i z_{k}}\left\langle x_{m_{k+1}, j}-x_{m_{k+1}, i z_{k}}, s\left(x_{m_{k+1}, i z_{k}}\right)-s\left(x_{m_{k+1}, j}\right)\right\rangle \\
& \leq 0
\end{aligned}
$$

which implies

$$
\left\langle x-x_{0}, s\left(x_{m_{k}, i}\right)\right\rangle \leq \frac{1}{z_{k}}\left\langle x-x_{0}, \sum_{j=i z_{k}}^{(i+1) z_{k}-1} s\left(x_{m_{k+1}, j}\right)\right\rangle .
$$

If $f$ is strictly convex, all these inequalities are strict, since then the subdifferential mapping is strictly monotone.

\section{A Characterization of $\varepsilon$-Subgradients}

Using Rockafellar integration formula, an expression for the $\varepsilon$-subdifferential operator of a lower semicontinuous proper convex function in terms of its subdifferential was given in [9, Theorem 1]. In this section we obtain a simpler characterization of $\varepsilon$-subgradients thanks to our new integration formula (1).

Theorem 2 Let $f$ and $s$ be as in Theorem $1, x \in \operatorname{dom} f, \varepsilon \geq 0$ and $x^{*} \in \mathbb{R}^{n}$. Then $x^{*} \in \partial_{\mathcal{E}} f(x)$ if and only if

$$
\left\langle x-x_{0}, x^{*}-\frac{1}{m} \sum_{i=0}^{m-1} s\left(x_{m, i}\right)\right\rangle \geq-\varepsilon \quad \forall x_{0} \in \operatorname{ridom} f, \forall m=1,2, \ldots
$$


Proof If $x^{*} \in \partial_{\varepsilon} f(x)$ and $x_{0} \in$ ri $\operatorname{dom} f$, then $f\left(x_{0}\right) \geq f(x)+\left\langle x_{0}-x, x^{*}\right\rangle-\varepsilon$ and $f(x) \geq f\left(x_{0}\right)+\frac{1}{m}\left\langle x-x_{0}, \sum_{i=0}^{m-1} s\left(x_{m, i}\right)\right\rangle$. By adding up these inequalities, simplifying and rearranging, we easily obtain (10). Conversely, assume that $x^{*}$ satisfies (10). Let $x_{0} \in \operatorname{ri} \operatorname{dom} f$ and $\alpha>f\left(x_{0}\right)-f(x)$. By (1), there exists $m$ such that $\frac{1}{m}\langle x-$ $\left.x_{0}, \sum_{i=0}^{m-1} s\left(x_{m, i}\right)\right\rangle>-\alpha$. Hence, by (10), we have $\varepsilon \geq\left\langle x-x_{0}, \frac{1}{m} \sum_{i=0}^{m-1} s\left(x_{m, i}\right)-x^{*}\right\rangle>$ $-\alpha-\left\langle x-x_{0}, x^{*}\right\rangle$. Since $\alpha$ is any number larger than $f\left(x_{0}\right)-f(x)$, it follows that $\varepsilon \geq-\left(f\left(x_{0}\right)-f(x)\right)-\left\langle x-x_{0}, x^{*}\right\rangle$, i.e., $f\left(x_{0}\right) \geq f(x)+\left\langle x_{0}-x, x^{*}\right\rangle-\varepsilon$. Since $f$, being convex, is upper semicontinuous along lines [12, Theorem 10.2], and the relative interior of any segment joining $x_{0}$ and a point in $\operatorname{dom} f$ is contained in ri $\operatorname{dom} f$, the preceding inequality also holds for an arbitrary $x_{0} \in \operatorname{dom} f$. This proves that $x^{*} \in \partial_{\varepsilon} f(x)$.

By means of the same argument used in the proof of Corollary 6, from Theorem 2 we obtain the following alternative characterization of $\varepsilon$-subgradients in terms of directional derivatives.

Corollary 8 Let $f, x, \varepsilon \geq 0$ and $x^{*}$ be as in Theorem 2. Then the following statements are equivalent:

(i) $x^{*} \in \partial_{\varepsilon} f(x)$.

(ii) One has

$$
\left\langle x-x_{0}, x^{*}-\frac{1}{m} \sum_{i=0}^{m-1} f^{\prime}\left(x_{m, i}, x-x_{0}\right)\right\rangle \geq-\varepsilon \quad \forall x_{0} \in \operatorname{ridom} f, \forall m=1,2, \ldots
$$

(iii) One has

$$
\left\langle x-x_{0}, x^{*}+\frac{1}{m} \sum_{i=0}^{m-1} f^{\prime}\left(x_{m, i},-\left(x-x_{0}\right)\right)\right\rangle \geq-\varepsilon \quad \forall x_{0} \in \operatorname{ridom} f, \forall m=1,2, \ldots
$$

Let us recall that a set is said to be nearly convex if it contains the relative interior of its convex hull.

Corollary 9 Let $f: \mathbb{R}^{n} \longrightarrow \mathbb{R} \cup\{+\infty\}$ be a l.s.c. proper convex function. Then $\partial f$ is maximal in the set of mappings $A: \mathbb{R}^{n} \rightrightarrows \mathbb{R}^{n}$ that have a nearly convex domain and a single valued selection $s$ on $\operatorname{Dom} A$ satisfying the following property:

$$
\begin{aligned}
& \left\langle x-x_{0}, x^{*}-\frac{1}{m} \sum_{i=0}^{m-1} s\left(x_{m, i}\right)\right\rangle \geq 0 \\
& \forall x \in \operatorname{Dom} A, \forall x_{0} \in \operatorname{ri} \operatorname{Dom} A, \forall x^{*} \in A(x), \forall m=1,2, \ldots
\end{aligned}
$$

Proof Since $\partial f$ is maximal monotone, its domain is nearly convex [10]. Moreover, property (11) is an easy consequence of the cyclic monotonicity of $\partial f$. Maximality immediately follows by applying Theorem 2 with $\varepsilon=0$. 


\section{Integration of $\varepsilon$-Subdifferentials of Functions Defined on Locally Convex} Spaces

In this section we consider the more general setting of locally convex spaces. Due to the lack of a suitable result on the nonemptiness of the subdifferential in this context, our results will be expressed in terms of $\varepsilon$-subdifferentials.

Theorem 3 Let $X$ be a real locally convex space with dual $X^{*}$ and duality pairing $\langle\cdot, \cdot\rangle: X \times X^{*} \longrightarrow \mathbb{R}$, let $f: X \longrightarrow \mathbb{R} \cup\{+\infty\}$ be a l.s.c. convex function, $x_{0} \in \operatorname{Dom} \partial f$, and $s: \operatorname{dom} f \times] 0,+\infty\left[\longrightarrow X^{*}\right.$ be such that $s(x, \varepsilon) \in \partial_{\varepsilon} f(x)$ for every $x \in \operatorname{dom} f$ and $\varepsilon>0$. Given $x \in \operatorname{dom} f$ for $m=2,3, \ldots$ and $i=0,1, \ldots, m$, denote $x_{m, i}:=x_{0}+\frac{i}{m}(x-$ $\left.x_{0}\right)$. If $\left\{\varepsilon_{m}\right\}$ is a sequence of strictly positive numbers such that $m \varepsilon_{m} \rightarrow 0$, then

$$
f(x)=f\left(x_{0}\right)+\lim _{m \rightarrow \infty} \frac{1}{m}\left\langle x-x_{0}, \sum_{i=1}^{m-1} s\left(x_{m, i}, \varepsilon_{m}\right)\right\rangle .
$$

Hence, for $x \in \operatorname{dom} f$ and $x^{*} \in \partial f\left(x_{0}\right)$, one has

$$
f(x)=f\left(x_{0}\right)+\sup _{m}\left\{\frac{1}{m}\left(\left\langle x-x_{0}, x^{*}+\sum_{i=1}^{m-1} s\left(x_{m, i}, \varepsilon_{m}\right)\right\rangle\right)-(m-1) \varepsilon_{m}\right\} .
$$

Proof The proof follows the same pattern as the proof of Theorem 1, with small changes. We have

$$
f\left(x_{m, i+1}\right)-f\left(x_{m, i}\right) \geq \frac{1}{m}\left\langle x-x_{0}, s\left(x_{m, i}, \varepsilon_{m}\right)\right\rangle-\varepsilon_{m} \quad \text { for } i=1, \ldots, m-1
$$

and, taking $x^{*} \in \partial f\left(x_{0}\right)$,

$$
f\left(x_{m, 1}\right)-f\left(x_{0}\right) \geq \frac{1}{m}\left\langle x-x_{0}, x^{*}\right\rangle .
$$

Adding up these inequalities, we get

$$
f(x)-f\left(x_{0}\right) \geq \frac{1}{m}\left\langle x-x_{0}, x^{*}+\sum_{i=1}^{m-1} s\left(x_{m, i}, \varepsilon_{m}\right)\right\rangle-(m-1) \varepsilon_{m},
$$

and hence

$$
f(x)-f\left(x_{0}\right) \geq \varlimsup_{m \rightarrow \infty} \frac{1}{m}\left\langle x-x_{0}, \sum_{i=1}^{m-1} s\left(x_{m, i}, \varepsilon_{m}\right)\right\rangle .
$$

On the other hand, we also have

$$
f\left(x_{m, i-1}\right)-f\left(x_{m, i}\right) \geq-\frac{1}{m}\left\langle x-x_{0}, s\left(x_{m, i}, \varepsilon_{m}\right)\right\rangle-\varepsilon_{m} \quad \text { for } i=1, \ldots, m-1,
$$

and adding up these inequalities we get

$$
f\left(x_{0}\right)-f\left(x_{m, m-1}\right) \geq-\frac{1}{m}\left\langle x-x_{0}, \sum_{i=1}^{m-1} s\left(x_{m, i}, \varepsilon_{m}\right)\right\rangle-(m-1) \varepsilon_{m}
$$


therefore, since $\underline{\lim }_{m \rightarrow \infty} f\left(x_{m, m-1}\right) \geq f(x)$ due to the lower semicontinuity of $f$,

$$
f\left(x_{0}\right)-f(x) \geq-\varliminf_{m \rightarrow \infty} \frac{1}{m}\left\langle x-x_{0}, \sum_{i=1}^{m-1} s\left(x_{m, i}, \varepsilon_{m}\right)\right\rangle .
$$

From (14) and (15), equality (12) imediately follows. To prove (13), we observe that the inequality " $\geq$ " is immediate, and obtain the opposite inequality using (12) together with the equality

$$
\begin{aligned}
\lim _{m \rightarrow \infty} & \frac{1}{m}\left\langle x-x_{0}, \sum_{i=1}^{m-1} s\left(x_{m, i}, \varepsilon_{m}\right)\right\rangle \\
& =\lim _{m \rightarrow \infty}\left(\frac{1}{m}\left\langle x-x_{0}, x^{*}+\sum_{i=1}^{m-1} s\left(x_{m, i}, \varepsilon_{m}\right)\right\rangle-(m-1) \varepsilon_{m}\right) .
\end{aligned}
$$

From Theorem 3, one can easily derive the following analogues of Corollaries 1 and 2 by mimicking their proofs.

Corollary 10 Let $X, f$ and $x_{0}$ be as in Theorem 3. Then, for every $x \in \operatorname{dom} f$ one has

$$
f(x)=f\left(x_{0}\right)+\sup _{\substack{\left(x_{i}, x_{i}^{*}\right) \in \operatorname{graph} \partial f_{\varepsilon} \\(i=1, \ldots, m) \\ \varepsilon>0}}\left\{\sum_{i=0}^{m-1}\left\langle x_{i+1}-x_{i}, x_{i}^{*}\right\rangle+\left\langle x-x_{m}, x_{m}^{*}\right\rangle-(m+1) \varepsilon\right\} .
$$

A formula very similar to (16) for the 1.s.c. convex hull of an epi-pointed function defined on a Banach space has been obtained by R. Correa, Y. García and A. Hantoute [5, Theorem 13].

Corollary 11 (see [4, Corollary 2.5]) Let $X$ be a real locally convex space and $f, g$ : $X \longrightarrow \mathbb{R} \cup\{+\infty\}$ be l.s.c. convex functions with a common domain $\operatorname{dom} f=\operatorname{dom} g=$ : $C$, and such that $\operatorname{Dom} \partial f \cap \operatorname{Dom} \partial g \neq \emptyset$ and

$$
\partial_{\varepsilon} f(x) \cap \partial_{\varepsilon} g(x) \neq \emptyset \quad \forall x \in C, \forall \varepsilon>0 .
$$

Then $f=g+$ const.

Acknowledgements I am very grateful to two anonymous referees for their helpful remarks; I feel particularly indebted to the one who pointed out that the intial version needed several important corrections.

\section{References}

1. Bachir, M., Daniilidis, A., Penot, J.-P.: Lower subdifferentiability and integration. Set-Valued Anal. 10, 89-108 (2002)

2. Benoist, J., Daniilidis, A.: Integration of Fenchel subdifferentials of epi-pointed functions. SIAM J. Optim. 12, 575-582 (2002)

3. Benoist, J., Daniilidis, A.: Subdifferential representation of convex functions: refinements and applications. J. Convex Anal. 12, 255-265 (2005) 
4. Burachik, R.S., Martínez-Legaz, J.E., Rocco, M.: On a sufficient condition for equality of two maximal monotone operators. Set-Valued Anal. 18, 327-335 (2010)

5. Correa, R., García, Y., Hantoute, A.: Integration formulas via the Fenchel subdifferential of nonconvex functions. Nonlinear Anal. 75, 1188-1201 (2012)

6. Hantoute, A., Martínez-Legaz, J.E.: Characterization of Lipschitz continuous difference of convex functions. J. Optim. Theory Appl. 159, 673-680 (2013).

7. Kocourek, P.: An elementary new proof of the determination of a convex function by its subdifferential. Optimization 59, 1231-1233 (2010)

8. López, M.A., Volle, M.: Subdifferential of the closed convex hull of a function and integration with nonconvex data in general normed spaces. J. Math. Anal. Appl. 390, 307-312 (2012)

9. Martínez-Legaz, J.E., Théra, M.: $\varepsilon$-Subdifferentials in terms of subdifferentials. Set-Valued Anal. 4, 327-332 (1996)

10. Minty, G.J.: On the maximal domain of a "monotone" function. Mich. Math. J. 8, 135-137 (1961)

11. Rockafellar, R.T.: Characterization of the subdifferentials of convex functions. Pac. J. Math. 17, 497510 (1966)

12. Rockafellar, R.T.: Convex Analysis. Princeton University Press, Princeton, NJ (1970)

13. Rockafellar, R.T., Wets, R.J.-B.: Variational Analysis. Springer-Verlag, Berlin (1998)

14. Verona, A., Verona, M.E.: Epiconvergence and $\varepsilon$-subgradients of convex functions. J. Convex Anal. 1, 87-100 (1994)

15. Zălinescu, C.: Convex Analysis in General Vector Spaces. World Scientific Publishing Co., Inc., River Edge, NJ (2002) 